\title{
Properties of quadratic equations and their application to power system analysis
}

\author{
Y.V. Makarov ${ }^{\mathrm{a}, 1}$, D.J. Hill ${ }^{\mathrm{b}, *}$, I.A. Hiskens ${ }^{\mathrm{c}, 2}$ \\ ${ }^{\text {a }}$ Southern Company Services Inc., 600 North 18th Street, PO Box 2625, Birmingham, AL 35202, USA \\ ${ }^{\mathrm{b}}$ School of Electrical and Information Engineering, The University of Sydney, Sydney, NSW 2006, Australia \\ ${ }^{\mathrm{c}}$ Department of Electrical and Computer Engineering, University of Illinois at Urbana-Champaign, 1406 West Green Street, Urbana, IL 61801, USA
}

Accepted 7 July 1999

\begin{abstract}
A number of facts about quadratic algebraic problems and applied Newton-Raphson like methods are presented. The main results are about solution structure, loading trajectories, load flow feasibility boundaries and Newton-Raphson solutions. Various practically important applications of these properties are discussed. Although motivated by power flow problems, the results are valid for any problem described by an algebraic system of quadratic equations. (C) 2000 Published by Elsevier Science Ltd. All rights reserved.
\end{abstract}

Keywords: Network analysis; Load flow analysis; Numerical techniques

\section{Introduction}

Different problems in power system analysis and control can be described by algebraic sets of quadratic equations of the form

$f(x)=y+g(x)=0$

where $x \in \mathbb{R}_{x}^{n}$ is a vector of unknown variables, $y \in \mathbb{R}_{y}^{n}$ is a vector of specified parameters, and $g$ is a quadratic function of $x$.

A classical example is the load flow problem where $x$ consists of nodal voltages, $y$ represents specified nodal parameters such as nodal powers, and $g$ is the nodal power mismatch function. There are two main forms used for the power mismatch equations: the polar and rectangular forms. Both of them have some advantages. The polar form provides significant reduction of computations. For instance, the method, which uses $\mathrm{P}-\mathrm{Q}$ decomposition of the load flow problem [1], is widely used in practice. The rectangular form of power flow equations can be effectively used as well-see Refs. [2-8]. The most important features of that form are that the power mismatch function can be exactly expressed using linear and second order terms of the

\footnotetext{
* Corresponding author. Tel.: +61-2-9351-4647; fax: +61-2-9351-5132.

E-mail address: davidh@ee.usyd.edu.au (D.J. Hill).

${ }^{1}$ Tel.: +1-205-257-6107; fax: +1-205-257-1040.

${ }^{2}$ Tel.: +1-217-333-1056; fax: +1-217-333-1162.
}

Taylor series, and its Jacobian matrix $J(x)=\partial f / \partial x$ is a linear function of active and reactive components of nodal voltages. Note that highly nonlinear static characteristics of loads can be always presented as quadratic functions by means of Taylor series approximations and introducing additional variables to reduce their nonlinearity.

The load flow problem is certainly not the only example of quadratic algebraic problems met in power system analysis. In fact, solutions of any optimisation problem with linear and quadratic objective function and constraints can be found by solving a quadratic system whose equations are obtained by differentiating the corresponding Lagrange function with respect to unknown variables and Lagrange multipliers. For instance, the problem of finding the shortest distance from a current operating point to the load flow feasibility boundary, which is often considered as the voltage collapse boundary, can be expressed as a quadratic task [9]. Once again, the problem can be represented as a quadratic equations set with the Jacobian matrix $J(x)$ being a linear function of unknown variables.

In any nonlinear problem, there are some important theoretical issues including solution existence, numerical method behaviour and their convergence to particular solutions, location of multiple solutions, configuration of singular boundaries where det $J(x)=0$, mutual arrangement of solutions and singular boundaries and solution behaviour in vicinity of singular boundaries. Such studies have already given valuable practical results. 
Studies of the multiple solutions of the load flow problem play a role in determining proximity to voltage collapse $[5,10]$. In order to obtain multiple load flow solutions, Tamura et al. used a set of quadratic load flow equations and the Newton-Raphson (NR) optimal multiplier method [7]. Iba et al. used Tamura's approach and some newly discovered convergence peculiarities of the NR method to find a pair of closest multiple solutions [8]. It is observed from experimental results [8] that if a point $x$ comes close to a line connecting a couple of distinct solutions, a further NR iterative process in rectangular form goes along this line. The next observation in Ref. [8] is that, in the vicinity of a singular point, the NR method with the optimal multiplier gives a trajectory which tends to the straight line connecting a pair of closely located but distinct solutions. These features are effectively used in Ref. [8] to locate multiple load flow solutions. The authors asked for a theoretical background of these experimentally discovered properties.

The present paper is written to further explore some fundamental properties of quadratic algebraic problems and use them in a number of practical applications. The main results establish the following properties:

- A variation of $x$ along a straight line through a pair of distinct solutions of the problem $f(x)=0$ results in variation of the mismatch vector $f(x)$ along a straight line in $\mathbb{R}_{y}^{n}$.

- There is a singular point in the middle of a straight line connecting a pair of distinct solutions $x_{1}, x_{2}$ in $\mathbb{R}_{x}^{n}[5,6,11$ and others].

- A vector co-linear to a straight line connecting a pair of distinct solutions in $\mathbb{R}_{x}^{n}$ nullifies the Jacobian matrix $J(x)=\partial f / \partial x$ at the centre point of the line [5,6 and others].

- If $x$ belongs to a straight line connecting a pair of distinct solutions, the NR iterative process goes along that line.

- The maximal number of solutions on any straight line in $\mathbb{R}_{x}^{n}$ is two.

- Along a straight line through two distinct solutions $x_{1}, x_{2}$, the problem can be reduced to a single scalar quadratic equation which locates these solutions.

- If a loading process $y(\beta)$ in $\mathbb{R}_{y}^{n}$ reaches a singular point $\operatorname{det} J(x)=0$, the corresponding trajectory of $x(\beta)$ in $\mathbb{R}_{x}^{n}$ tends to the right eigenvector nullifying $J(x)$ at the singular point (except in some special cases).

- At any singular point, there are two merging solutions (except in some special cases).

- For any two points $x_{1} \neq x_{2}$ and the Jacobian matrix $\operatorname{det} J\left(x_{1}\right) \neq 0$, the number and location of singularities of the quadratic problem $f(x)=0$ on the straight line through $x_{1}, x_{2}$ is defined by real eigenvalues of the matrix $J^{-1}\left(x_{1}\right) J\left(x_{2}\right)$.

Some of the results are already known-see references above. They are assembled here with new results and clearer explanations. Earlier versions of the results presented here are given in Refs. [2,12,13].

\section{Quadratic power flow studies}

This section deals with some basic properties of the quadratic power flow problems, their solutions, singularities, and the NR method.

\subsection{Property 1}

\subsubsection{Formulation}

For a quadratic problem $f(x)=0$, there is a point of singularity in the centre of a straight line connecting a pair of distinct solutions in $\mathbb{R}_{x}^{n}$, and a vector co-linear to this line nullifies the Jacobian matrix evaluated in the centre point.

\subsubsection{Proof}

Let $x_{1}, x_{2}$ be two distinct solutions of a quadratic problem $f(x)=0$. A line connecting these solutions can be defined by

$x=x_{1}+\mu\left(x_{2}-x_{1}\right)=x_{1}+\mu \Delta x_{21}$

where $\mu$ is a parameter, and $\Delta x_{21}=x_{2}-x_{1}$. Due to quadratic nonlinearity, we have

$f\left(x_{1}\right)=f\left(x_{2}\right)-J\left(x_{2}\right) \Delta x_{21}+0.5 W\left(-\Delta x_{21}\right)$

$f\left(x_{2}\right)=f\left(x_{1}\right)+J\left(x_{1}\right) \Delta x_{21}+0.5 W\left(\Delta x_{21}\right)$

where $0.5 W\left(\Delta x_{21}\right)$ is the quadratic term of the Taylor series expansion, Eq. (4). It is clear that $W(-\Delta x)=W(\Delta x)$. From Eqs. (3) and (4),

$\left[J\left(x_{1}\right)+J\left(x_{2}\right)\right] \Delta x_{21}=0$

For a quadratic function $f(x)$, the Jacobian matrix contains elements which are linear functions of $x$. So, it can be represented as

$J(x)=\sum_{i=1}^{n} A_{i} x_{i}+J(0)$

where $A_{i}, J(0)$ are $(n \times n)$ constant matrices of Jacobian coefficients, $x_{i} \in x$. Using Eq. (6), the equality (5) can be rewritten as

$2 J\left(x_{0}\right) \Delta x_{21}=0$

where $x_{0}=\left(x_{1}+x_{2}\right) / 2$. As $\Delta x_{21} \neq 0$, the vector $\Delta x_{21}$ is the right eigenvector corresponding to a zero eigenvalue of the Jacobian matrix. Moreover, for all $x \neq 0$, which are colinear vectors with respect to $\Delta x_{21}$, we get $J\left(x_{0}\right) x=0$.

\subsubsection{Comments}

Both the first and second parts were proved in Refs. [2,5,11 and others]. The above proof seems to be more simple and compact.

\subsubsection{Examples}

Let us take an example of the New England Test System [14]. The system consists of 39 buses, 10 generators, and 18 
Table 1

Distinct load flow solutions

\begin{tabular}{|c|c|c|c|c|c|c|}
\hline \multirow[t]{2}{*}{ Bus } & \multicolumn{2}{|c|}{ Solution $x_{1}$} & \multicolumn{2}{|c|}{ Solution $x_{2}$} & \multicolumn{2}{|c|}{ Solution $x_{3}$} \\
\hline & $\mathrm{V}, \mathrm{kV}$ & $\delta, \operatorname{grad}$ & $\mathrm{V}, \mathrm{kV}$ & $\delta, \operatorname{grad}$ & $\mathrm{V}, \mathrm{kV}$ & $\delta, \operatorname{grad}$ \\
\hline 1 & 103.1 & -24.9 & 0.0 & -191.2 & 89.3 & -42.4 \\
\hline 2 & 101.7 & -18.6 & 86.9 & -11.0 & 64.1 & -41.1 \\
\hline 3 & 95.5 & -20.4 & 92.0 & -15.1 & 3.8 & -125.2 \\
\hline 4 & 84.2 & -19.6 & 94.6 & -15.7 & 44.5 & -39.2 \\
\hline 5 & 77.0 & -16.4 & 96.6 & -14.1 & 64.0 & -29.0 \\
\hline 6 & 78.7 & -15.0 & 97.3 & -13.1 & 67.7 & -26.4 \\
\hline 7 & 68.5 & -19.2 & 96.0 & -16.3 & 66.3 & -32.7 \\
\hline 8 & 64.1 & -20.4 & 95.9 & -17.3 & 66.5 & -34.5 \\
\hline 9 & 0.0 & 104.0 & 100.8 & -23.4 & 88.9 & -40.2 \\
\hline 10 & 88.2 & -12.9 & 98.9 & -10.8 & 73.5 & -25.3 \\
\hline 11 & 84.9 & -13.6 & 98.2 & -11.6 & 71.2 & -25.7 \\
\hline 12 & 84.6 & -13.9 & 96.8 & -11.7 & 67.6 & -26.7 \\
\hline 13 & 87.9 & -14.1 & 98.2 & -11.7 & 68.9 & -27.5 \\
\hline 14 & 87.9 & -16.9 & 96.9 & -13.6 & 59.0 & -34.3 \\
\hline 15 & 94.2 & -18.7 & 97.5 & -14.5 & 64.3 & -45.0 \\
\hline 16 & 98.6 & -17.7 & 99.4 & -13.1 & 69.5 & -45.2 \\
\hline 17 & 98.3 & -19.1 & 97.2 & -14.3 & 52.3 & -49.7 \\
\hline 18 & 97.1 & -20.1 & 95.1 & -15.1 & 32.0 & -56.4 \\
\hline 19 & 103.5 & -12.9 & 103.8 & -8.4 & 93.6 & -39.1 \\
\hline 20 & 98.3 & -14.2 & 98.5 & -9.7 & 92.6 & -40.8 \\
\hline 21 & 100.0 & -15.1 & 100.6 & -10.6 & 79.5 & -41.2 \\
\hline 22 & 103.4 & -10.5 & 103.7 & -6.0 & 92.5 & -35.1 \\
\hline 23 & 102.7 & -10.7 & 103.1 & -6.2 & 91.5 & -35.3 \\
\hline 24 & 99.5 & -17.5 & 100.3 & -13.0 & 73.0 & -45.1 \\
\hline 25 & 103.5 & -17.3 & 93.9 & -12.0 & 75.6 & -45.8 \\
\hline 26 & 102.4 & -17.9 & 98.3 & -12.9 & 73.4 & -48.1 \\
\hline 27 & 99.9 & -19.7 & 97.2 & -14.9 & 62.5 & -51.7 \\
\hline 28 & 103.6 & -14.3 & 101.5 & -9.2 & 88.8 & -43.2 \\
\hline 29 & 104.1 & -11.5 & 102.7 & -6.3 & 94.1 & -39.9 \\
\hline 30 & 104.8 & -16.2 & 104.8 & -8.3 & 104.8 & -37.4 \\
\hline 31 & 98.2 & -0.0 & 98.2 & 0.0 & 98.2 & 0.0 \\
\hline 32 & 98.3 & -4.8 & 98.3 & -3.7 & 98.3 & -15.6 \\
\hline 33 & 99.7 & -8.3 & 99.7 & -3.8 & 99.7 & -34.4 \\
\hline 34 & 101.2 & -9.1 & 101.2 & -4.6 & 101.2 & -35.5 \\
\hline 35 & 104.9 & -5.7 & 104.9 & -1.2 & 104.9 & -29.7 \\
\hline 36 & 106.4 & -2.7 & 106.4 & 1.7 & 106.4 & -26.52 \\
\hline 37 & 102.8 & -10.7 & 102.8 & -4.9 & 102.8 & -37.3 \\
\hline 38 & 102.7 & -4.7 & 102.7 & 0.5 & 102.7 & -32.7 \\
\hline 39 & 103.0 & -28.6 & 103.0 & -27.3 & 103.0 & -42.5 \\
\hline
\end{tabular}

loads. Bus number 31 is chosen as a slack bus. All other generators are represented by means of constant active powers and terminal voltages. Loads have fixed active and reactive demands. We consider three distinct load flow solutions $x_{1}, x_{2}$ and $x_{3}$ given in Table 1 . These solutions were selected from a solution set obtained by the method for computing multiple solutions of quadratic algebraic problems given in Section 3. All three points are low voltage solutions. As the points $x_{1}, x_{2}$ and $x_{3}$ do not belong to a straight line, they define a plane in $\mathbb{R}_{x}^{n}$ which we call the $\Delta$-plane. The $\Delta$-plane is very convenient for viewing the mutual arrangement of solutions and singularities of a load flow problem [15]. Actually, solutions $x_{1}, x_{2}$ and $x_{3}$ can be shown as points in the plane-see Fig. 1. Straight lines connecting these three solutions belong to the planethey are drawn as dashed lines. The cut-set of the feasibility domain boundary where $\operatorname{det} J(x)=0$ by the $\Delta$-plane is given by dotted curves. To get the cut-set, the X-ray theorem and $\Delta$-plane method were used-see Section 3 and Ref. [15]. In full compliance with Property 1, the singular points marked by small circles lie exactly in the middle of the dashed lines connecting the points $x_{1}, x_{2}$ and $x_{3}$.

\subsection{Property 2}

\subsubsection{Formulation}

For quadratic mismatch functions $f(x)$, a variation of $x$ along a straight line through a pair of distinct solutions of the problem $f(x)=0$ results in variation of the mismatch vector $f(x)$ along a straight line in $\mathbb{R}_{y}^{n}$.

\subsubsection{Proof}

Let $x$ be a point on the straight line connecting two distinct solutions $x_{1}, x_{2}$ described by Eq. (2). For quadratic mismatch functions,

$$
\begin{aligned}
& f(x)=f\left(x_{1}\right)+\mu J\left(x_{1}\right) \Delta x_{21}+0.5 \mu^{2} W\left(\Delta x_{21}\right) \\
& f\left(x_{2}\right)=f\left(x_{1}\right)+J\left(x_{1}\right) \Delta x_{21}+0.5 W\left(\Delta x_{21}\right)
\end{aligned}
$$

where $0.5 W\left(\Delta x_{21}\right)$ is the quadratic term of the Taylor series expansion, Eq. (9). At points $x_{1}, x_{2}$, we have $f\left(x_{1}\right)=f\left(x_{2}\right)=$ 0. So, from Eq. (9),

$0.5 W\left(\Delta x_{21}\right)=-J\left(x_{1}\right) \Delta x_{21}$

Using Eq. (10), Eq. (8) transforms to

$f\left(x_{1}+\mu \Delta x_{21}\right)=\mu(1-\mu) J\left(x_{1}\right) \Delta x_{21}=\beta \Phi$

where $\beta=\mu(1-\mu), \Phi=\mathrm{J}\left(\mathrm{x}_{1}\right) \Delta \mathrm{x}_{21}$. Thus the mismatch function $f\left(x_{1}+\mu \Delta x_{21}\right)$ varies along the straight line $\beta \Phi$ in $\mathbb{R}_{y}^{n}$.

\subsubsection{Comments}

This fact was mentioned in Ref. [5]. An interesting conclusion follows from Properties 1 and 2. Variations of $x$ along a straight line connecting a couple of distinct solutions are actually motions of $x$ along the right eigenvector nullifying $J(x)$ in the middle of the line.

\subsubsection{Examples}

In the same New England test system and for the same solution set $x_{1}, x_{2}$ and $x_{3}$, let us consider a map $y=-y_{0}-$ $g(x)$ of the $\Delta$-plane in Fig. 1 into the space $\mathbb{R}_{y}^{n}$-see Fig. 2. Point 0 represents the condition $y_{0}+g\left(x_{i}\right)=0, x_{i}=$ $x_{1}, x_{2}, x_{3}$. As the straight lines defined by $x_{1}\left(\mu_{1}\right)=$ $x_{1}+\mu_{1} \Delta x_{21}$ and $x_{2}\left(\mu_{2}\right)=x_{1}+\mu_{2} \Delta x_{31}$ are mapped into the distinct straight lines $y_{1}\left(\mu_{1}\right)=\mu_{1}\left(1-\mu_{1}\right) J\left(x_{1}\right) \Delta x_{21}$ and $y_{2}\left(\mu_{2}\right)=\mu_{2}\left(1-\mu_{2}\right) J\left(x_{1}\right) \Delta x_{31}$ which intersect at point 0 , the last two lines define a plane in $\mathbb{R}_{y}^{n}$. This plane is referred to as the $\Delta$-plane in $\mathbb{R}_{y}^{n}$. All points which belong to the straight lines $x_{1}\left(\mu_{1}\right)$ and $x_{2}\left(\mu_{2}\right)$ are given accurately as the corresponding points on the lines $y_{1}\left(\mu_{1}\right)$ and $y_{2}\left(\mu_{2}\right)$ including all singular points with $\operatorname{det} J(x)=0$. Singular 


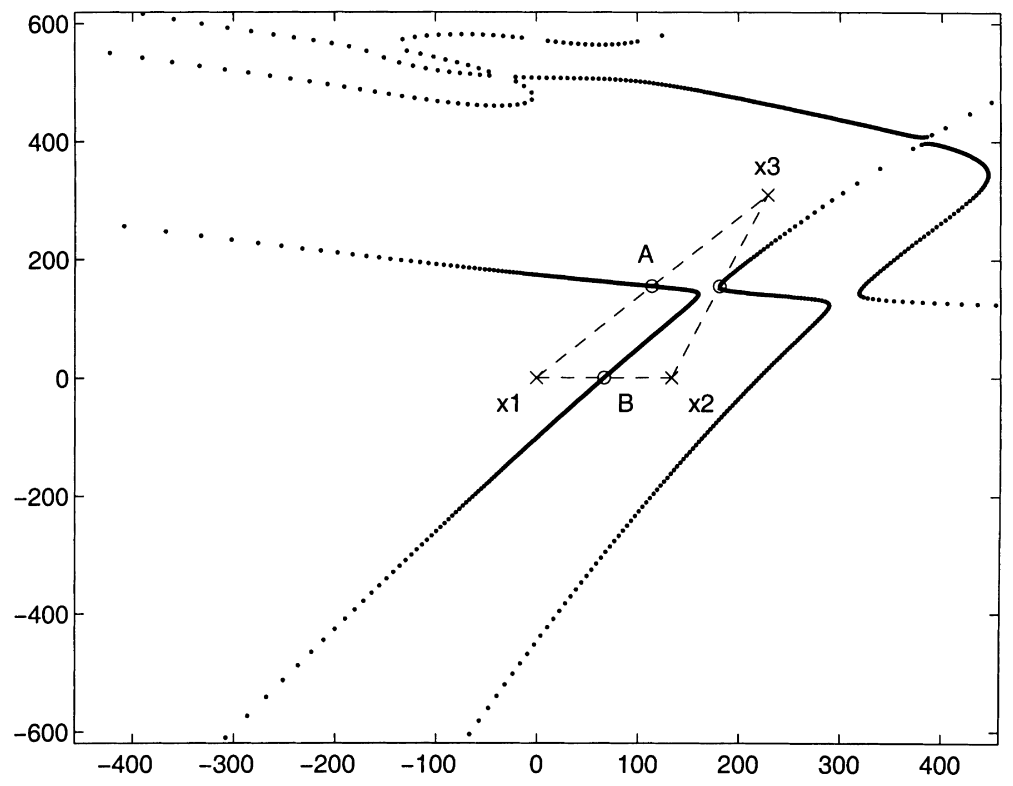

Fig. 1. $\Delta$-plane in $\mathbb{R}_{x}^{n}$ (New England Test System).

points $A$ and $B$ correspond to the points $A$ and $B$ in Fig. 1. They have a specific nature. For example, a loading process $x_{1}\left(\mu_{1}\right)$ results in a straight motion from point 0 to point $A, \mu_{1}<1 / 2$. At point $A$, the maximum loadability point with $\mu_{1}=1 / 2$ is reached. A further loading along $x_{1}\left(\mu_{1}\right), \mu_{1}>1 / 2$, results in backward straight motion from $A$ to 0 . All other singular points (dotted curves beyond the straight lines $0-A$ and $0-B$ ) are obtained as projections of multidimensional singular curves on the $\Delta$-plane in $\mathbb{R}_{y}^{n}$.

\subsection{Property 3}

\subsubsection{Formulation}

If any point $x$ is on a straight line connecting two distinct solutions of the quadratic problem $f(x)=0$, the NewtonRaphson iterative process with the initial point from $x$ follows this line.

\subsubsection{Proof}

If any point $x^{i}$ is on the line connecting two distinct

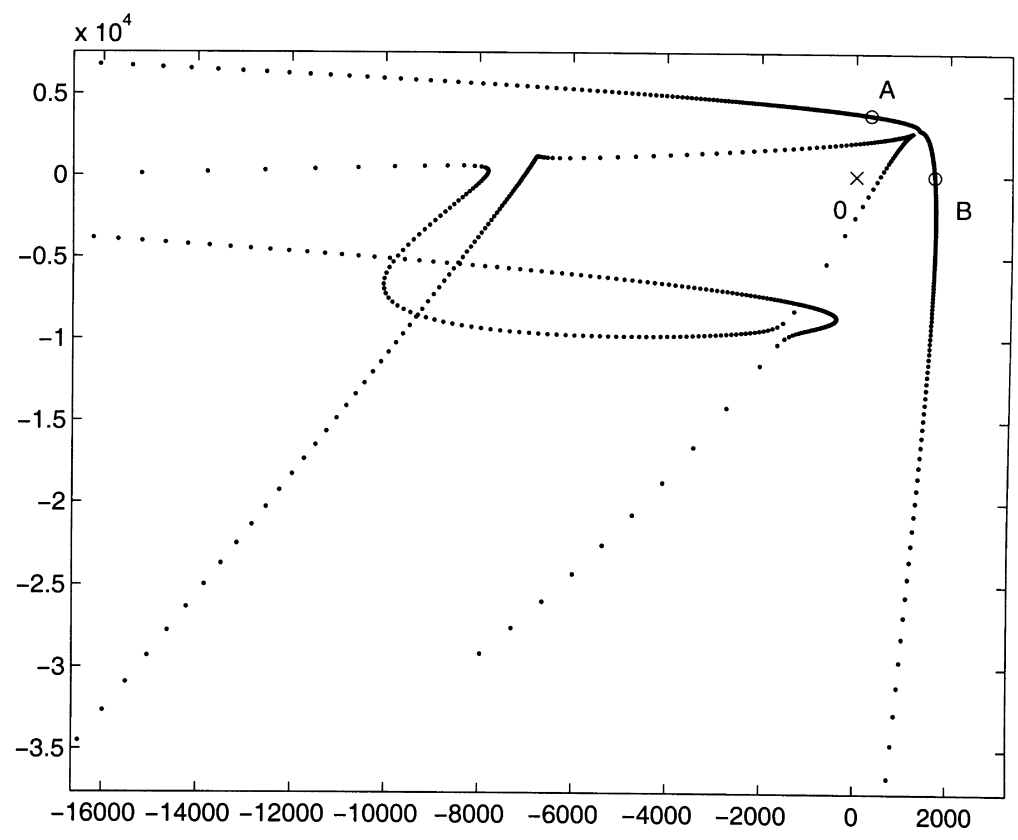

Fig. 2. $\Delta$-plane in $\mathbb{R}_{y}^{n}$ (New England Test System). 


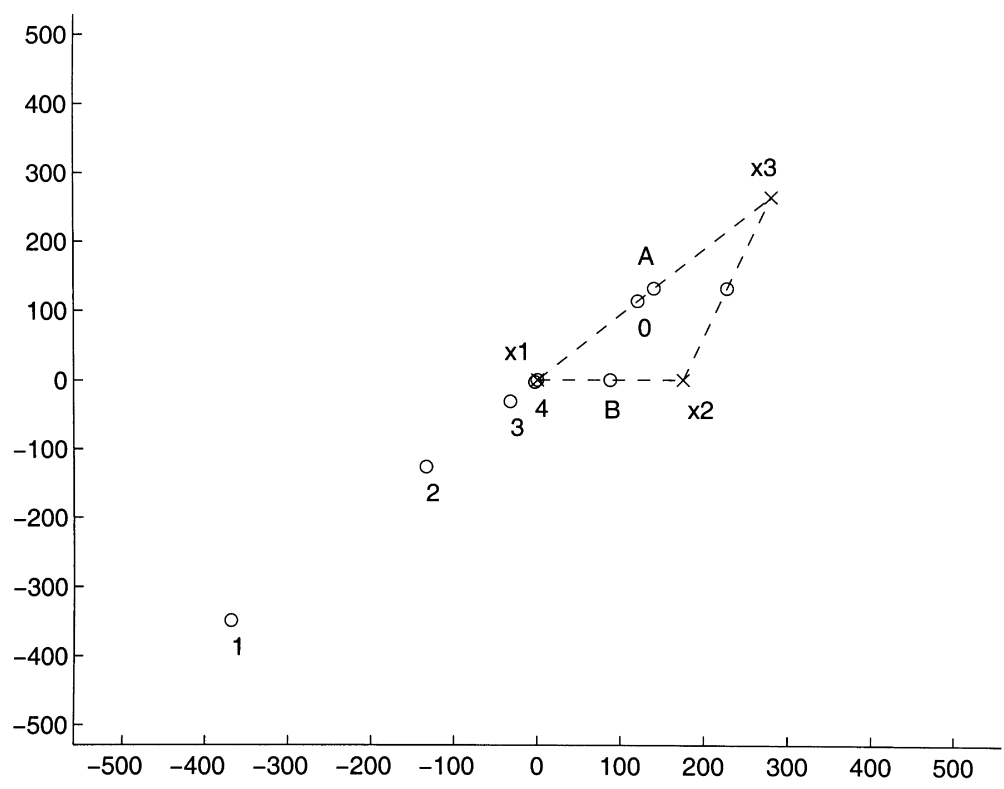

Fig. 3. Newton-Raphson process along the line connecting two distinct solutions (New England Test System).

solutions it can be described in the form (2). The middle point of $\Delta x_{21}$ is $x_{0}=0.5\left(x_{1}+x_{2}\right)$. The quadratic mismatch function can be expressed as

$f\left(x^{i}\right)=f\left(x_{0}\right)+J\left(x_{0}\right)\left(x^{i}-x_{0}\right)+0.5 W\left(x^{i}-x_{0}\right)$

To express the last term of Eq. (12) in terms of Jacobian matrices, we write

$f(\Delta x)=f(0)+J(0) \Delta x+0.5 W(\Delta x)$

$f(0)=f(\Delta x)-J(\Delta x) \Delta x+0.5 W(\Delta x)$

By summing of the last two equalities,

$W(\Delta x)=[J(\Delta x)-J(0)] \Delta x$,

and so

$0.5 W\left(x^{i}-x_{0}\right)=0.5\left[J\left(x^{i}-x_{0}\right)-J(0)\right]\left(x^{i}-x_{0}\right)$

On the contrary, taking into account the quadratic nonlinearity of $f(x)$ and Eq. (6),

$J\left(x^{i}-x_{0}\right)=J\left(x^{i}\right)-J\left(x_{0}\right)+J(0)$

Therefore, in Eq. (12), we have

$0.5 W\left(x^{i}-x_{0}\right)=0.5\left[J\left(x^{i}\right)-J\left(x_{0}\right)\right]\left(x^{i}-x_{0}\right)$

Noting Eq. (7), then $J\left(x_{0}\right)\left(x^{i}-x_{0}\right)=0$. From Eq. (12),

$f\left(x^{i}\right)=f\left(x_{0}\right)+0.5 J\left(x^{i}\right)\left(x^{i}-x_{0}\right)$

For the NR method with initial point $x^{i}$, we have the following expression for the correction vector $\Delta x^{i}$

$f\left(x^{i}\right)+J\left(x^{i}\right) \Delta x^{i}=0$

From Eq. (11),

$f\left(x^{i}\right)=\mu(1-\mu) J\left(x_{1}\right) \Delta x_{21}$ and so

$J\left(x_{1}\right) \Delta x_{21}=\mu^{-1}(1-\mu)^{-1} f\left(x^{i}\right), \quad \mu \neq 0,1$

At the point $\mu=0.5$, we have $x^{i}=x_{0}$, and it follows from Eqs. (17) and (18) that

$f\left(x_{0}\right)=0.25 \mu^{-1}(1-\mu)^{-1} f\left(x^{i}\right)$

By the substitution of Eq. (19) into Eq. (15), it follows

$f\left(x^{i}\right)=0.25 \mu^{-1}(1-\mu)^{-1} f\left(x^{i}\right)+0.5 J\left(x^{i}\right)\left(x^{i}-x_{0}\right)$

Multiplying Eq. (20) by $J^{-1}\left(x^{i}\right)$ and taking into account Eq. (16),

$\Delta x^{i}=-2 \mu(1-\mu)[4 \mu(1-\mu)-1]^{-1}\left(x^{i}-x_{0}\right)$

Eq. (21) shows that the NR correcting vector $\Delta x^{i}$ belongs to the straight line directed by the vector $\left(x^{i}-x_{0}\right)$, i.e. the iterative process goes along the line connecting $x_{1}, x_{2}$.

\subsubsection{Comments}

This fact was experimentally discovered in Ref. [8], where again the authors asked for some theoretical proof of the phenomena.

\subsubsection{Examples}

In the same New England test system, let us consider Newton-Raphson iterations starting from the point $x=$ $x_{1}+0.43\left(x_{3}-x_{1}\right)$ which lies exactly on the line $\left(x_{1}-x_{3}\right)$-see point 0 in Fig. 3. A numerical simulation has been done, and it has been discovered that the entire NR process goes along the same line on the $\Delta$-plane-see points 1, 2, 3 and 4 in Fig. 3. The initial leap from point 0 to point 1 is explained by the fact that the point 0 is taken rather close to the singular point $A$. 


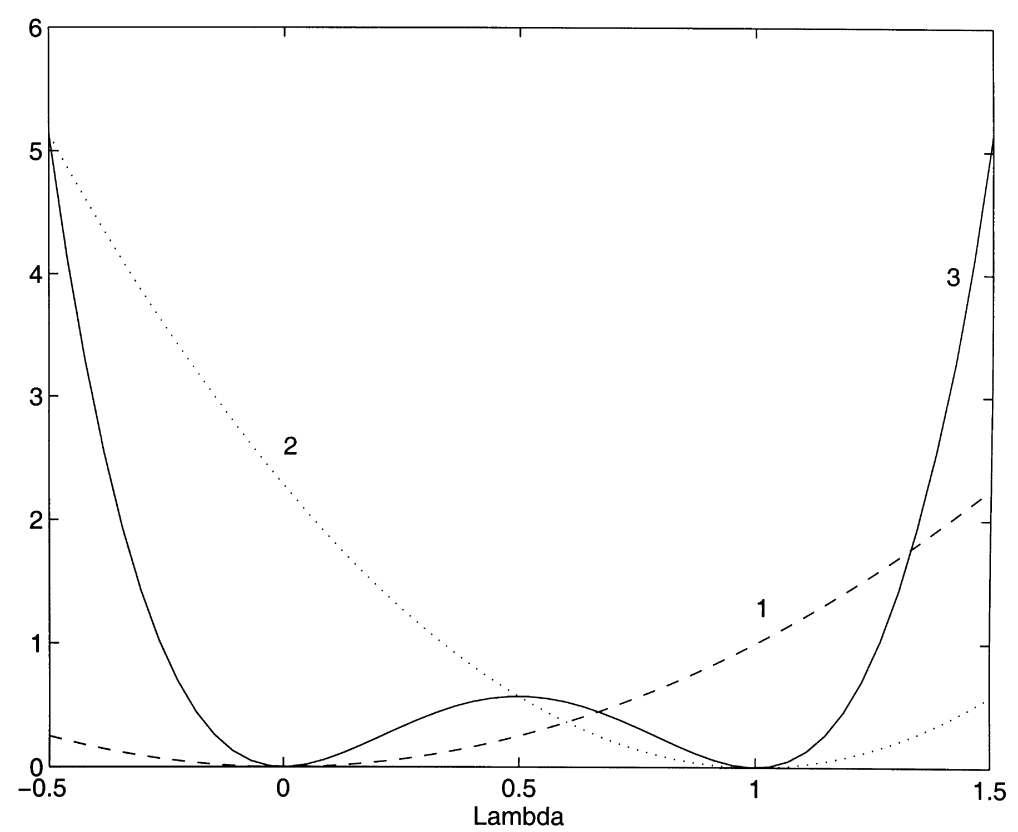

Fig. 4. Two distinct solutions along the connecting line (New England Test System): 1-Curve $\lambda^{2} ; 2$-Curve $a \lambda^{2}+b \lambda+c ; 3-C u r v e ~ \phi(x *, \lambda)=\left(a \lambda^{2}+b \lambda+\right.$ c) $\lambda^{2}$.

\subsection{Property 4}

\subsubsection{Formulation}

The maximum number of solutions of a quadratic equation $f(x)=0$ on each straight line in the state space $\mathbb{R}_{\infty}^{n}$ is two.

\subsubsection{Proof}

Let us take the function

$\phi(\lambda, x)=f^{\mathrm{t}}(x+\lambda \Delta x) f(x+\lambda \Delta x)$

For a quadratic mismatch function $f(x)$,

$f(x+\lambda \Delta x)=f(x)+\lambda J(x) \Delta x+0.5 \lambda^{2} W(\Delta x)$

So,

$$
\begin{aligned}
& \phi(\lambda, x)=\left\|f(x)+\lambda J(x) \Delta x+0.5 \lambda^{2} W(\Delta x)\right\|^{2} \\
& =\|f(x)\|^{2}+\|\lambda J(x) \Delta x\|^{2}+\left\|0.5 \lambda^{2} W(\Delta x)\right\|^{2} \\
& \quad+2 \lambda f^{\mathrm{t}}(x) J(x) \Delta x+\lambda^{3} W^{\mathrm{t}}(x) J(x) \Delta x+\lambda^{2} f^{\mathrm{t}}(x) W(\Delta x)
\end{aligned}
$$

The function $\phi(\lambda, x)$ equals zero if and only if $f(x+\lambda \Delta x)=$ 0 . At a solution point $x=x_{*}, f\left(x_{*}\right)=0$,and the function (22) is

$$
\begin{aligned}
\phi\left(\lambda, x_{*}\right)= & 0.25 \lambda^{4}\|W(\Delta x)\|^{2}+\lambda^{3} W^{\mathrm{t}}(\Delta x) J\left(x_{*}\right) \Delta x \\
& +\lambda^{2}\left\|J\left(x_{*}\right) \Delta x\right\|^{2} \\
= & \left(a \lambda^{2}+b \lambda+c\right) \lambda^{2}
\end{aligned}
$$

where $a, b, c$ are the obvious functions of $\Delta x$. For any fixed direction $\Delta x \neq 0, \phi\left(\lambda, x_{*}\right)$ equals zero in the two following cases
(a) $\lambda=0$;
(b) $a \lambda^{2}+b \lambda+c=0$.

The first case gives us the original solution point $x=x_{*}$. The second case corresponds to solutions $x \neq x_{*}$ on the straight line directed by $\Delta x$. However, as it is clear from Eq. (22), that function (22) cannot be negative. Thus $a \lambda^{2}+$ $b \lambda+c \geq 0$, and in case (b) it is possible to have only one additional solution except $x_{*}$, but not two or more. So, on the line we get one root $x=x_{*}$, and we can have only one additional root corresponding to condition (b).

\subsubsection{Examples}

Fig. 4 shows a typical shape of the scaled functions $\lambda^{2}$ (curve 1), $a \lambda^{2}+b \lambda+c \quad\left(\right.$ curve 2) and $\phi\left(\lambda, x_{*}\right)=$ $\left(a \lambda^{2}+b \lambda+c\right) \lambda^{2}$ (curve 3) along a straight line $x_{*}+\lambda \Delta x$ connecting two distinct solutions in the New England test system. Two zero minima of $\phi\left(\lambda, x_{*}\right)$ at $\lambda=0$ and $\lambda=1$ correspond to the first solution $x_{*}$ and additional solution $x \neq x_{*}$

\subsection{Property 5}

\subsubsection{Formulation}

For a straight line, connecting two solutions in $\mathbb{R}_{x}^{n}$, the system of quadratic equations can be reduced to a single scalar quadratic equation, which locates these solutions.

\subsubsection{Proof}

Let $x_{1}, x_{2}$ be unknown distinct solutions of a quadratic 


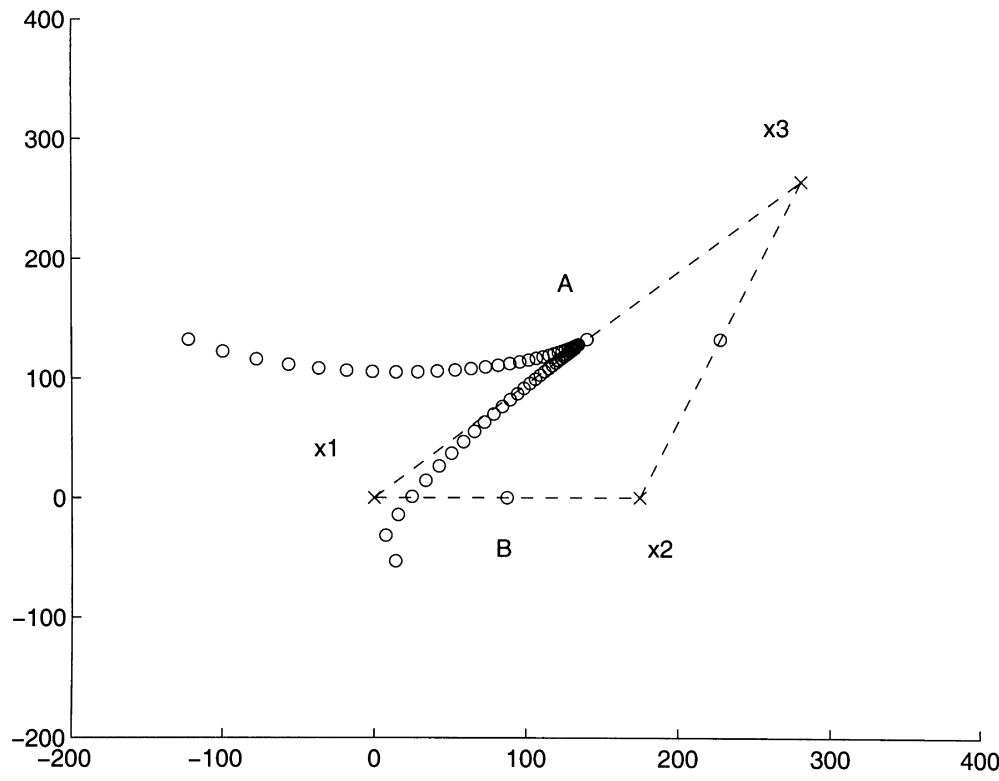

Fig. 5. Two loading trajectories $x(\beta)$ ending at singular point $A$ (New England Test System).

problem $f(x)=0$. Suppose we have a point $x_{*}$ and direction $\Delta x_{*}$ which define a line connecting the pair of solutions. The mismatch function calculated along this line is

$F(\alpha)=f\left(x_{*}+\alpha \Delta x_{*}\right)$

where $\alpha$ is a scalar parameter. Let us take any fixed value $\alpha=\alpha_{*}$ and define a constant

$\Phi_{*}=F\left(\alpha_{*}\right) \neq 0$

Having Eqs. (23) and (24), consider the equation

$\Phi_{*}^{\mathrm{t}} F(\alpha)=0$

It follows from Property 1 that $\Phi_{*}$ and $F(\alpha)$ are co-linear vectors, and Eq. (25) is true only if $F(\alpha)=0$. Using Eq. (24) and the Taylor series expansion

$F(\alpha)=f\left(x_{*}\right)+\alpha J\left(x_{*}\right) \Delta x_{*}+0.5 \alpha^{2} W\left(\Delta x_{*}\right)$

we get the scalar quadratic equation

$a \alpha^{2}+b \alpha+c=0$

where $a=0.5 \Phi_{*}^{\mathrm{t}} W\left(\Delta x_{*}\right), \quad b=\Phi_{*}^{\mathrm{t}} J\left(x_{*}\right) \Delta x_{*}, c=\Phi_{*}^{\mathrm{t}} f\left(x_{*}\right)$. Eq. (26) has a pair of distinct real roots $\alpha_{1}, \alpha_{2}$ corresponding to $x_{1}, x_{2}$, and we can define these solutions as $x_{1}=$ $x_{*}+\alpha_{1} \Delta x_{*}, x_{2}=x_{*}+\alpha_{2} \Delta x_{*}$.

\subsection{Property 6}

\subsubsection{Formulation}

For almost all cases, if any loading process $y(\beta)$ ends at a singular point $x_{0}$ of the problem $y(\beta)+g(x)=0$, where $g(x)$ is a quadratic function of $x$ and $\beta$ is a scalar loading parameter, there are two distinct solutions $x_{1}, x_{2}$ merging at the singular point, and the trajectories $x_{1}(\beta), x_{2}(\beta)$ tend to the right eigenvector $r$ corresponding to a zero eigenvalue of the Jacobian matrix $J\left(x_{0}\right)$.

\subsubsection{Proof}

Let a loading process with $\beta$ variable and

$y(\beta)+g(x)=0$

end at a singular point $x_{0}$ corresponding to $\beta=\beta_{0}$. At the singular point, $\mathrm{d} y=y_{\beta}^{\prime}\left(\beta_{0}\right) \mathrm{d} \beta=Y_{0} \mathrm{~d} \beta$. The implicit function theorem gives

$J\left(x_{0}\right) \mathrm{d} x+\mathrm{d} y=J\left(x_{0}\right) \mathrm{d} x+Y_{0} \mathrm{~d} \beta=0$

By multiplying Eq. (28) by $s^{\mathrm{t}}$, where $s$ is the left eigenvector of $J\left(x_{0}\right)$ corresponding to a zero eigenvalue, we get

$s^{\mathrm{t} J} J\left(x_{0}\right) \mathrm{d} x+s^{\mathrm{t}} Y_{0} \mathrm{~d} \beta=s^{\mathrm{t}} Y_{0} \mathrm{~d} \beta=0$

For the general case of loading, $s^{t} Y_{0} \neq 0$, and, therefore, $\mathrm{d} \beta=0$. This, means that the loading parameter $\beta$ reaches its extremal value $\beta_{0}$ at the singular point $x_{0}$. Alternatively, when $s^{t} Y_{0}=0$, the loading trajectory $y(\beta)$ tends to the tangent hyper-plane to the singular margin $(\operatorname{det} J(x)=0)$ of the problem $f(x)=0$ at the point $x_{0}$. It follows from the fact that $s$ is an orthogonal vector with respect to the singular margin in $\mathbb{R}_{y}^{n}$.

Using Eq. (28), we have

$J\left(x_{0}\right) \mathrm{d} x=0$

So, the increment $\mathrm{d} x$ has the same direction as the right eigenvector $r$ of the Jacobian matrix corresponding to its zero eigenvalue, and the last part of Property 6 has been proved.

On the contrary, having Eq. (29),

$-Y_{0} \Delta \beta=J\left(x_{0}\right) \Delta x+\frac{1}{2} W(\Delta x) \stackrel{\Delta \beta \rightarrow 0}{\rightarrow} \frac{1}{2} W(\Delta x)$ 
and $W(\Delta x)=W(-\Delta x)$. So, for a small increment $\Delta \beta$ at the point $x_{0}$, we have two increments of $x$ of opposite signs directed along $r$. Therefore, the first part of Property 6 has been proved.

\subsubsection{Comments}

The property follows from [16, p. 217]. A similar fact was mentioned in Ref. [17]. The proof presented here was first given in Ref. [9]. It explains the experimental fact obtained in Ref. [8]. It was observed that in the vicinity of a singular point the NR method with the optimal multiplier gives a trajectory which tends to the straight line connecting a pair of closely located load flow solutions $x_{1}, x_{2}$. Actually, the loading trajectory $y(\beta)$ in Eq. (27) can be represented as the convergence trajectory of the NR method. If it comes close to the singular margin, it tends to the right eigenvector $r$, which nullifies the Jacobian matrix in the middle point between closely located solutions. Property 3 says that the further iterative process goes along the line directed by the vector $\left(x_{1}, x_{2}\right)$, and that the line is co-linear to $r$. So, Properties 3 and 6 explain these phenomena.

\subsubsection{Examples}

Fig. 5 shows two curves $x(\beta)$ (circled curves) corresponding to two different loading trajectories $y(\beta)$ ending at singular point $A$. It is seen that both the trajectories $x(\beta)$ tend to the right eigenvector $r=x_{2}-x_{1}$ which nullifies the Jacobian matrix at point $A$.

\subsection{Property 7 (X-ray theorem)}

\subsubsection{Formulation}

For any two points $x_{1} \neq x_{2}$ and det $J\left(x_{1}\right) \neq 0$, the number and location of singularities of the quadratic problem $f(x)=$ 0 on the straight line through $x_{1}, x_{2}$ is defined by real eigenvalues of the matrix $J^{-1}\left(x_{1}\right) J\left(x_{2}\right)$.

\subsubsection{Proof}

Let us define the line through $x_{1}, x_{2}$ as Eq. (2). Using Eq. (6), it is easy to show that

$J\left[x_{1}+\mu\left(x_{2}-x_{1}\right)\right]=(1-\mu) J\left(x_{1}\right)+\mu J\left(x_{2}\right)$

As $x_{1}$ is a nonsingular point, for $\mu \neq 0$, expression (30) can be written as

$J(x)=\mu J\left(x_{1}\right)\left[J^{-1}\left(x_{1}\right) J\left(x_{2}\right)-(\mu-1) \mu^{-1} I\right]$

where $I$ is the identity matrix. Therefore, all singular points on the line, Eq. (2) can be computed as real eigenvalues of the matrix $J^{-1}\left(x_{1}\right) J\left(x_{2}\right)$.

\subsubsection{Comments}

The X-ray theorem can be effectively used to locate all singularities of the load flow Jacobian matrix along a given ray in the space of dependent variables $x$. In particular, it forms a basis of the $\Delta$-plane method given in Section 3.

\section{Power system applications}

This section illustrates how the properties analysed in Section 2 can be used in power system analysis.

\subsection{Finding multiple solutions of a quadratic problem}

Property 1 allows to find multiple solutions of a quadratic problem $f(x)=0$, for example, the load flow problem. Note that Eq. (11) can be rewritten as

$f\left(x_{1}+\Delta x\right)+(\mu-1) J\left(x_{1}\right) \Delta x=0$

where $x_{1}$ is a known solution, $\Delta x$ an unknown increment of state variables and $\mu$ an unknown scalar parameter. Except the trivial case $\Delta x=0$, the last equation corresponds to a different solution

$x_{2}=x_{1}+\mu^{-1} \Delta x, \quad|\mu|<\infty, \quad \mu \neq 0$

System (31) has $n$ equations and $n+1$ unknown variables, so it is necessary to add an additional equation in Eq. (31), for instance,

$r^{\mathrm{t}} \Delta x-1=0$

where $r$ is a nonzero vector. By varying $r$ and substitution of newly discovered solutions instead of $x_{1}$ in Eqs. (31) and (32), it is possible to get all solutions of a quadratic problem. Tables 1 contains some of the distinct solutions in the New England test system. The total number of solutions obtained exceeds 70 .

\section{2. $\Delta$-plane method}

The X-ray theorem allows us to develop a new robust method for finding the power system load flow feasibility boundary and, more generally speaking, the singular boundaries of the power flow problem on the $\Delta$-plane defined by any three vectors of dependent variables (nodal voltages). An advantage of the method is that it does not require an iterative solution of nonlinear equations (except the eigenvalue problem). Besides benefits for visualisation and fully flexible observation, the method is a useful tool for topological studies of power system multiple solution structures and stability domains. For instance, any three equilibria (load flow solutions) can be chosen to form the $\Delta$-plane, and the relative positions of these points and bifurcation surfaces will be reflected on the $\Delta$-plane in all details.

\subsubsection{Obtaining bifurcation curves on the $\Delta$-plane}

A plane in $\mathbb{R}_{x}^{n}$ can be defined by any three distinct points $x_{1}, x_{2}$ and $x_{3}$ provided the condition

$x_{3} \neq x_{1}+\mu\left(x_{2}-x_{1}\right)$

is satisfied for any scalar parameter $\mu$. Condition (33) means that the points $x_{1}, x_{2}$ and $x_{3}$ do not belong to a straight line in $\mathbb{R}_{x}^{n}$. Once Eq. (33) is true, the points $x_{1}, x_{2}$ and $x_{3}$ form a triangular $\Delta$, which defines a plane in $\mathbb{R}_{x}^{n}$ and gives the name for the proposed method. Any point on the $\Delta$-plane 
can be expressed by means of scalar parameters $\gamma_{1}$ and $\gamma_{2}$ :

$x\left(\gamma_{1}, \gamma_{2}\right)=x_{1}+\gamma_{1}\left(x_{2}-x_{1}\right)+\gamma_{2}\left(x_{3}-x_{1}\right)$

It is clear that $x(0,0)=x_{1}, x(1,0)=x_{2}$ and $x(0,1)=x_{3}$. Suppose that at the point $x_{1}$, det $J\left(x_{1}\right) \neq 0$. Then the following procedure can be used to find out all singularities of $J(x)$ in the $\Delta$-plane. The idea consists in rotation of a vector $x\left(\gamma_{1}, \gamma_{2}\right)-x_{1}$ in the $\Delta$-plane, and subsequent computations of all singularities of $J(x)$ on each line defined by this vector. Let $\theta$ be the angle, which defines the current orientation of the vector $x\left(\gamma_{1}, \gamma_{2}\right)-x_{1}$ with respect to the vector $x_{2}-x_{1}$ in the $\Delta$-plane. The following steps are used:

1. Choose the angle $\theta=0$.

2. Compute $\gamma_{1}=l \cos \theta$ and $\gamma_{2}=l \sin \theta, l \gg 0$.

3. Define a point $x=x\left(\gamma_{1}, \gamma_{2}\right)$ as in Eq. (34).

4. Find eigenvalues of the matrix $J^{-1}\left(x_{1}\right) J(x)$.

5. Compute $\mu_{j}=\left(1-\lambda_{j}\right)^{-1}$ for all real eigenvalues $\lambda_{j} \neq 1$ from the previous step.

6 . For each value of $\mu_{j}$, define the corresponding point in the $\Delta$-plane as

$$
x_{j}(\theta)=x_{1}+\mu_{j}\left[\gamma_{1}\left(x_{2}-x_{1}\right)+\gamma_{2}\left(x_{3}-x_{1}\right)\right]
$$

7. Change $\theta=\theta+\Delta \theta$, where $\Delta \theta$ is an increment, and go to step 2 unless $\theta \geq \pi$.

The set of points $x_{j}(\theta)$ computed for different $\theta$ forms a cut-set of the feasibility domain boundary by the $\Delta$ plane. The procedure does not require an iterative solution except as needed for the eigenvalue problem. The reliable QR technique is recommended to be used in step 4

\subsubsection{Visualisation of the $\Delta$-plane in $\mathbb{R}_{x}^{n}$}

Although the bifurcation points $x_{j}(\theta)$ in Eq. (35) belong to the $\Delta$-plane, they are vectors in the multidimensional space $\mathbb{R}_{x}^{n}$. To get a visual representation for them, it is convenient to use a new two-dimensional coordinate system associated with the $\Delta$-plane itself. For this purpose, we use the following oblique-angled coordinate system:

$\tilde{x}_{1}=\left[\begin{array}{ll}0 & 0\end{array}\right]^{\mathrm{t}}$

$\tilde{x}_{2}=\left\|x_{2}-x_{1}\right\|\left[\begin{array}{ll}1 & 0\end{array}\right]^{\mathrm{t}}$

$\tilde{x}_{3}=\left\|x_{3}-x_{1}\right\|\left[\begin{array}{cc}\cos \alpha_{x} & \sin \alpha_{x}\end{array}\right]^{\mathrm{t}}$

where

$\alpha_{x}=\arccos \frac{\left(x_{3}-x_{1}\right)^{\mathrm{t}}\left(x_{2}-x_{1}\right)}{\left\|x_{3}-x_{1}\right\|\left\|x_{2}-x_{1}\right\|}$

In the new two-dimensional coordinate system, the expression for computing the power flow singular points becomes the following:

$\tilde{x}_{j}(\theta)=\mu_{j}\left(\gamma_{1} \tilde{x}_{2}+\gamma_{2} \tilde{x}_{3}\right)$

\subsection{3. $\Delta$-plane in $\mathbb{R}_{y}^{n}$}

Consider a particular case when $x_{1}, x_{2}$ and $x_{3}$ are distinct solutions of the load flow problem. It follows from Property 2 in Section 2 that these points cannot lie on a straight line in $\mathbb{R}_{y}^{n}$. Property 3 says that, from $y=$ $-g(x)$, the straight lines

$x\left(\gamma_{1}, 0\right)=x_{1}+\gamma_{1}\left(x_{2}-x_{1}\right)$

$x\left(0, \gamma_{2}\right)=x_{1}+\gamma_{2}\left(x_{3}-x_{1}\right)$

in $\mathbb{R}_{x}^{n}$ are mapped into the straight lines

$y_{1}=y\left(\gamma_{1}, 0\right)=\gamma_{1}\left(\gamma_{1}-1\right) J\left(x_{1}\right)\left(x_{2}-x_{1}\right)$

$y_{2}=y\left(0, \gamma_{2}\right)=\gamma_{2}\left(\gamma_{2}-1\right) J\left(x_{1}\right)\left(x_{3}-x_{1}\right)$

in $\mathbb{R}_{y}^{n}$, respectively. The last two lines pass through a common point $-g\left(x_{1}\right)$, and they define a plane which we call the $\Delta$-plane in $\mathbb{R}_{y}^{n}$.

\subsubsection{Visualisation of the $\Delta$-plane in $\mathbb{R}_{y}^{n}$}

From $y=-g(x)$, all singular points computed along the lines (38) and (39) are mapped into points of the lines (40) and (41), respectively. So, they lie on the $\Delta$-plane in the space $\mathbb{R}_{y}^{n}$. The rest of the points $y_{j}(\theta)$ mapped from the $\Delta$ plane in $\mathbb{R}_{x}^{n}$ do not normally belong to the $\Delta$-plane in $\mathbb{R}_{y}^{n}$; the $\Delta$-plane in $\mathbb{R}_{x}^{n}$ is mapped by $y=-g(x)$ into a surface which is not a plane. The only thing, which we can do here, is to find out their projections $\tilde{y}_{j}(\theta)$ on the $\Delta$-plane in the space $\mathbb{R}_{y}^{n}$. The projection $\tilde{y}_{j}(\theta)$ can be found by the following way. Let

$\tilde{y}_{j}(\theta)=\beta_{1} \tilde{y}_{1}+\beta_{2} \tilde{y}_{2}$

where $\beta_{1}$ and $\beta_{2}$ are parameters, and $\tilde{y}_{1}, \tilde{y}_{2}$ are defined as

$\tilde{y}_{1}=\left\|y_{1}\right\|\left[\begin{array}{ll}1 & 0\end{array}\right]^{\mathrm{t}}$

$\tilde{y}_{2}=\left\|y_{2}\right\|\left[\begin{array}{cc}\cos \alpha_{y} & \sin \alpha_{y}\end{array}\right]^{\mathrm{t}}$

where $\alpha_{y}=\arccos \left\|y_{2}\right\|^{-1}\left\|y_{1}\right\|^{-1}\left(y_{2}\right)^{\mathrm{t}}\left(y_{1}\right)$. The coefficients $\beta_{1}$ and $\beta_{2}$ can be found by solving the linear equation

$\left(\begin{array}{cc}\left\|y_{1}\right\|^{2} & y_{1}^{\mathrm{t}} y_{2} \\ y_{1}^{\mathrm{t}} y_{2} & \left\|y_{2}\right\|^{2}\end{array}\right)\left(\begin{array}{l}\beta_{1} \\ \beta_{2}\end{array}\right)=\left(\begin{array}{c}y_{1}^{\mathrm{t}} y_{j}(\theta) \\ y_{2}^{\mathrm{t}} y_{j}(\theta)\end{array}\right)$

They are then used in Eq. (42) to get singular points in the two-dimensional oblique-angled coordinate system $\left(\tilde{y}_{1}, \tilde{y}_{2}\right)$

As the singular points belong to a nonlinear surface, and we use their projections on the $\Delta$-plane, the resulting plot reflects a qualitative shape of the singular boundary in $\mathbb{R}_{y}^{n}$. Nevertheless, all singular points along the lines $A B$ and $A C$ are defined accurately. 


\subsubsection{Examples}

The proposed method has been tested for the above New England Test System. We consider three distinct load flow solutions $x_{1}, x_{2}$ and $x_{3}$ given in Table 1. Solutions $x_{1}, x_{2}$ and $x_{3}$ define a $\Delta$-plane in $\mathbb{R}_{x}^{n}$ shown in Fig. 1 . The plane is oriented in such a way that $x_{1}$ corresponds to the zero point $(0,0)$, and the vector $x_{2}-x_{1}$ is directed along the horizontal axis. Solution $x_{1}$ is taken as a fixed point for the plot: all straight lines, along which the singularities are determined, belong to the $\Delta$-plane and pass through $x_{1}$. The cut-set of the feasibility domain boundary by the $\Delta$ plane is shown by dotted curves (solid parts of the curves are formed by covering of close dots).

A detailed consideration of the feasibility boundary topology is not the main purpose of this paper. Nevertheless, let us make some observations. Firstly, note that the singular points marked by small circles lie exactly in the middle of the dashed lines connecting the points $x_{1}, x_{2}$ and $x_{3}$. This fact is explained in a number of works-see Refs. [13,18], for example. Secondly, the singular curves in Fig. 1 have an open shape. This appears to say that, by certain unrestricted variations of independent parameters $y$ in $\mathbb{R}_{y}^{n}$, voltages $x$ in $\mathbb{R}_{x}^{n}$ may be indefinitely increased without reaching the feasibility boundary. In more realistic situations the limitations for $y$, for example, equality constraints for generator terminal voltages and zero power injections in empty buses, must be taken into account.

Fig. 2 shows the corresponding $\Delta$-plane in $\mathbb{R}_{y}^{n}$. Point 0 represents the operating condition $y_{0}+g(x)=0$ of the system. The singular boundary is plotted by using the map $y=-y_{0}-g(x)$. Points $A$ and $B$ correspond to the points $A$ and $B$ in Fig. 1. All singular points which belong to the straight lines defined by $0-A$ and $0-B$ are given accurately. All other points are obtained as projections of multidimensional singular curves on the $\Delta$-plane in $\mathbb{R}_{y}^{n}$.

\section{Conclusions}

In this paper, we have carefully studied the properties of quadratic equations with respect to solution structure, loading trajectories, load flow feasibility boundaries and NewtonRaphson solutions. These results were shown to clarify techniques used in power system analysis. This is explored for finding multiple solutions and bifurcation curves.

\section{Acknowledgements}

This work was sponsored in part by the Australian Electricity Supply Industry Research Board grant "Voltage Collapse Analysis and Control". The authors wish to thank Professors N. Barabanov (Russia), I. Dobson (USA), A. Volberg (USA, France) and V. Yakubovich (Russia) for their interest, examination and consultations regarding some aspects of the work. The authors also express their gratitude to Mr Bhudjanga Chakrabarti, who helped with the bibliography and put very valuable questions, which actually initiated the present work, and Mr Zhao-Yang Dong for assistance in the final preparation.

\section{References}

[1] Stott B, Alsac O. Fast decoupled load flow. IEEE Trans Power App Syst 1974;PAS-93(3):859-69.

[2] Kontorovich AM. A method of load flow and steady-state stability analysis for complicated power systems with respect to frequency variations. PhD thesis. Leningrad Polytechnic Institute, Leningrad, 1979 (in Russian).

[3] Iwamoto S, Tamura Y. A load flow calculation method for ill-conditioned power systems. Proceedings of the IEEE PES Summer Meeting. Vancouver, British Columbia, Canada, July 1979.

[4] Iwamoto S, Tamura Y. A load flow calculation method for ill-conditioned power systems. IEEE Trans Power App Syst 1981;PAS100(4):1736-43.

[5] Tamura Y, Sakamoto K, Tayama Y. Voltage instability proximity index (VIPI) based on multiple load flow solutions in ill-conditioned power systems. Proceedings of the 27th Conference on Decision and Control. Austin, Texas, December 1988.

[6] Tamura Y, Nakanishi Y, Iwamoto S. On the multiple solution structure, singular point and existence condition of the multiple load flow solutions. Proceedings of the IEEE PES Winter Meeting. New York, February 1980.

[7] Tamura Y, Iba K, Iwamoto S. A method for finding multiple loadflow solutions for general power systems. Proceedings of the IEEE PES Winter Meeting. New York, February 1980.

[8] Iba K, Suzuki H, Egawa M, Watanabe T. A method for finding a pair of multiple load flow solutions in bulk power systems. Proceedings of the IEEE Power Industry Computer Application Conference. Seattle, Washington, May 1989.

[9] Makarov YV, Hiskens IA. A continuation method approach to finding the closest saddle node bifurcation point. Proceedings of the NSF/ ECC Workshop on Bulk Power System Voltage Phenomena III Davos, Switzerland, Fairfax, Virginia: ECC Inc, 1994.

[10] Tamura Y, Mori H, Iwamoto S. Relationship between voltage instability and multiple load flow solutions in electric power systems. IEEE Trans Power App Syst 1983;PAS-102(5).

[11] Idelchik VI, Lazebnik AI. An analytical research of solution existence and uniqueness of electrical power system load flow equations. Izvestia AN SSSR: Energetika i Transport 1972;(2): 18-24 (in Russian).

[12] Makarov YV, Hiskens IA. Solution characteristics of the quadratic power flow problem. Technical Report No EE9377 (revised). Department of Electrical and Computer Engineering, University of Newcastle, Australia, May 1994.

[13] Makarov YV, Hiskens IA, Hill DJ. Study of multisolution quadratic load flow problems and applied Newton-Raphson like methods. Proceedings of the IEEE International Symposium on Circuits and Systems. Seattle, Washington, April/May 1995, paper No. 541.

[14] Byerly RT, Sherman DE, Bennon RJ. Frequency domain analysis of low frequency oscillations in large electric power systems. EPRI EL726. RP744-1 Interim Report. Palo Alto, California, April 1978.

[15] Makarov YV, Hill DJ, Dong ZY. A new robust method to explore the load flow feasibility boundaries. Proceedings of the Australasian Universities Power Engineering Conference AUPEC'96, vol. 1. Melbourne Australia, October 2-4, 1996. p. 137-42.

[16] Chow SN, Hale J. Methods of bifurcation theory, New York: Springer, 1982 Section 6.2. 
[17] Dobson I, Lu L. Voltage collapse precipated by the immediate change in stability when generator reactive power limits are encountered. IEEE Trans Circuits Systems-Fundamental Theory and Applications 1992;39(9):762-6.
[18] Makarov YV, Kontorovich AM, Hill DJ, Hiskens IA. Solution characteristics of quadratic power flow problems. Proceedings of the Power System Computation Conference, vol. 1. Dresden, Germany, 19-23 August, 1996. p. 460-7. 